
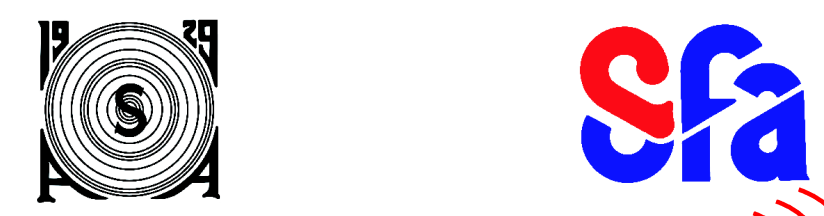

EAA
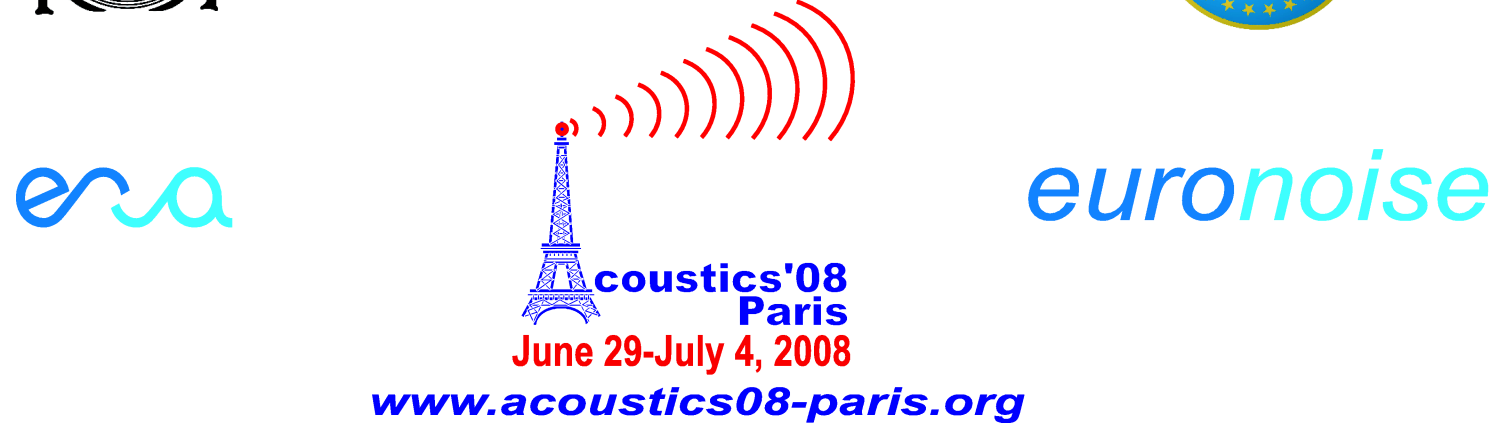

wWw.acoustics08-paris.org

\title{
Estimating the frequency dependent behavior of marine sediment sound speeds using low frequency aircraft sound
}
A. Amiri-Simkooei ${ }^{\mathrm{a}}$, M. Snellen ${ }^{\mathrm{a}}, \mathrm{D}$.
D. G Sim
ons $^{\mathrm{b}}$ and M. Buckingham ${ }^{\mathrm{c}}$

${ }^{a}$ Acoustic Remote Sensing Group, Delft Institute of Earth Observation and Space Systems, Delft University of Technology, Kluyverweg 1, 2629 HS Delft, Netherlands

${ }^{\mathrm{b}}$ Delft University of Technology, P.O. Box Postbus 5048, 2600 GA Delft, Netherlands ${ }^{\mathrm{c}}$ Marine Physical Lab, Scripps Institution of Oceanography, UCSD, 9500 Gilman Dr. M/C 0238, La Jolla, CA 92093-0238, USA

a.amirisimkooei@tudelft.nl 
To investigate the dispersion behavior of sediment sound speeds, broadband sound speed measurements are required. Sediment sound speed dispersion models predict the largest sound speed variations typically at low frequencies. Aircraft flyover noise acquired on receivers placed in sediment proved to be a promising approach towards broadband low-frequency measurements. Since the Doppler shift depends on sound speed at the receiver position, it can be used to derive the sound speed. We propose a new approach using the entire time series instead of employing only the two observed frequencies at approaching and departing. It is based on a model for received signals, accounting for the Doppler shifted frequency. By maximizing the match between modeled and measured time series as a function of sound speed and frequency in a least-squares framework, estimates of the frequency-dependent sound speed are obtained. The method is applied to data acquired at receivers placed in air, water and sediment. Statistical tests indicate that the marine sediment sound speed depends indeed on frequency, i.e. sound speed decreases significantly below $300 \mathrm{~Hz}$. A comparison is made with a modified viscous fluid model from which realistic values for the geotechnical parameters of the sediment were obtained.

\section{Introduction}

In Ref. [1] a novel approach is presented in which sound speeds at receiver positions in air, water and sediment, are estimated using airplane flyover noise. The method employs the Doppler shifts as observed in the received noise. Since the magnitude of the Doppler frequency shift depends on the sound speed at the receiver position, the observed Doppler shift can be used to derive values for the sound speed. Fourier analysis is employed to determine the Doppler shifted frequencies when the airplane is far away and approaching the receiver, and when it is far away and departing from the receiver. In [1] expressions are presented where the prevailing sound speed can directly be derived from the difference between the two above mentioned frequencies.

Here we propose an alternative approach. Instead of employing the frequencies at time instants where the airplane is at a large distance from the receivers, here the entire time series will be employed. A model for the received time series is adapted. By maximizing the match between modeled and measured time series as a function of sound speed and frequency, an estimate of sound speed as a function of frequency is obtained. The method is applied to data acquired at receivers placed in air, water and sediment as provided by [2]. The resulting curves for the receiver placed in the sediment are then compared with model outcomes. The model employed is described by [3] and predicts the sediment sound speed dispersion behavior as a function of sediment geotechnical parameters.

\section{Methodology}

\subsection{Optimization problem}

We aim to determine the sound speed in the air, water, and sediment for different frequencies using a leastsquares power spectrum density (PSD) function. The PSD is a function of two arguments: the frequency and the sound speed in the media. A two dimensional search method in which the power spectrum achieves it maximum value is presented.

Assume that we are given the time series $y=$ $\left[y_{1}, \ldots, y_{m}\right]^{T}$, which is the power of the received sound at a receiver placed in the air, water, or sediment (a zero-mean time series). To determine the sound speed in a medium for an individual frequency $\omega_{j}$, we write the time series $y$ as an individual trigonometric term

$$
\mathrm{E}\left(y\left(t_{i}\right)\right)=a_{j} \cos \omega_{j}^{d} t_{i}+b_{j} \sin \omega_{j}^{d} t_{i}, i=1, \ldots, m
$$

where $\mathrm{E}$ is the expectation operator, $y\left(t_{i}\right)=y_{i}$ is the observable at time instant $t_{i}, \omega_{j}^{d}=\omega_{j}^{d}(t)$ is the Doppler shifted frequency of the original frequency $\omega_{j}$ ( $t$ is measured in the receiver time frame - see Eq. 8), and $a_{j}$ and $b_{j}$ are unknown coefficients. This expression indicates that the observations are expressed as a sinusoidal wave in which its frequency changes over time due to the Doppler effect. The relation between $\omega_{j}$ and $\omega_{j}^{d}$ is

$$
\omega_{j}^{d}=\frac{\omega_{j}}{1-\frac{v}{c_{n}} \cos \theta_{n}}, n=1,2,3
$$

where $v$ is the speed of the aircraft in the horizontal, $c_{n}, n=1,2,3$ are the sound speeds in the three media considered, and $\theta_{n}$ are the corresponding elevation angles at the time of transmission ([1]); see Fig. 1.

Let us now consider a single medium (air, water or sediment), with $c_{j}$ being the sound speed in that medium at frequency $\omega_{j}$. We can rewrite Eq. (1) in a matrix form as $\mathrm{E}(y)=A_{j}^{d} x_{j}$, where

$$
A_{j}^{d}=\left[\begin{array}{cc}
\cos \omega_{j}^{d} t_{1} & \sin \omega_{j}^{d} t_{1} \\
\cos \omega_{j}^{d} t_{2} & \sin \omega_{j}^{d} t_{2} \\
\vdots & \vdots \\
\cos \omega_{j}^{d} t_{m} & \sin \omega_{j}^{d} t_{m}
\end{array}\right] \text { and } x_{j}=\left[\begin{array}{c}
a_{j} \\
b_{j}
\end{array}\right]
$$

The frequencies $\omega_{j}$ and sound speeds $c_{j}$ (and correspondingly $A_{j}^{d}$ ) are obtained by solving the following minimization problem:

$$
\omega_{j}, c_{j}=\arg \min _{\omega_{j}, c_{j}}\left\|y-A_{j}^{d} x_{j}\right\|^{2}=\arg \min _{\omega_{j}, c_{j}}\left\|P_{A_{j}^{d}}^{\perp} y\right\|^{2}
$$

where $\|\|=.\left((.)^{T}(.)\right)^{1 / 2}$ is the norm of a vector, and $P_{A_{j}^{d}}^{\perp}=I-A_{j}^{d}\left(A_{j}^{d T} A_{j}^{d}\right)^{-1} A_{j}^{d T}$ is an orthogonal projector. The above minimization problem is equivalent to the following maximization problem [4]:

$$
\omega_{j}, c_{j}=\arg \max _{\omega_{j}, c_{j}} y^{T} A_{j}^{d}\left(A_{j}^{d T} A_{j}^{d}\right)^{-1} A_{j}^{d T} y
$$

Therefore, for each frequency $\omega_{j}$ and sound speed $c_{j}$ considered we can determine the power spectrum of the zero-mean time series using the identity

$$
P\left(\omega_{j}, c_{j}\right)=y^{T} A_{j}^{d}\left(A_{j}^{d T} A_{j}^{d}\right)^{-1} A_{j}^{d T} y
$$


which conforms Eq. (5) and should be maximized over the frequency of the sound and the sound speed in the medium considered. Given an arbitrary frequency $\omega_{j}$, the sound speed can be obtained using the following maximization problem

$$
c_{j}=\arg \max _{c_{j}} y^{T} A_{j}^{d}\left(A_{j}^{d T} A_{j}^{d}\right)^{-1} A_{j}^{d T} y
$$

Analytical evaluation of this maximization is complicated. In practice, one has to be satisfied with numerical evaluation. A plot of spectral values $P\left(\omega_{j}, c_{j}\right)$ versus a set of discrete values for $c_{j}$ is used as a tool to investigate the contribution of different sound speeds in the construction of the observed time series. In other words, for each (discrete) frequency $\omega_{j}$ the power spectrum $P\left(\omega_{j}, c_{j}\right)$ is obtained as a function of the discrete values for $c_{j}$. The $c_{j}$ value for which the power spectrum achieves its maximum is considered to be an estimate for the sound speed in the medium considered.

In an ideal case where the original frequencies $\omega_{j}$ of harmonic functions are known (for example when the exact propeller frequency and its harmonics are known), the search method is limited to find the sound speeds for those frequencies. This however does not hold in reality. In practice, one has to be satisfied with a set of high-power frequencies around the original frequencies.

\subsection{Doppler shifted frequency}

Since the data set is measured in the receiver time frame, i.e. time of reception $t$ at the microphone or hydrophone, it is important to reformulate the expression of $\omega_{j}^{d}$ accordingly. For the measurements at the microphone, the Doppler shifted frequency given in Eq. (2) can be expressed as ([1])

$$
\omega_{j}^{d}=\frac{\omega_{j}}{1-(v / c)^{2}}\left(1-\frac{v^{2} t}{c_{1} \sqrt{(v t)^{2}}+(v / c)^{2} h_{1}^{2}}\right)
$$

where $h_{1}$ is the altitude of the aircraft above the microphone, and $t$ is measured in the receiver time frame such that $t=0$ is the time at which the aircraft is exactly above the receiver. This equation shows how the Doppler shifted frequency $\omega_{j}^{d}$ (of original frequency $\omega_{j}$ ) changes over time $t$. To obtain the sound speed in air, we will have to assume the terms $v, h_{1}$ and $t=0$ to be known. Therefore, any error in these parameters will directly affect our estimate.

For the water and sediment such a closed form expression does not exist. We will solve for the angles $\theta_{2}$ and $\theta_{3}$, used in Eq. (2), by an iterative Gauss-Newton method. In order to relate the time of reception $t$ to the angle $\theta_{w}$ at the air-water interface (see Fig. 1), and then $\theta_{2}$ and $\theta_{3}$ at the water or sediment phone, respectively, we derived a series of expressions based on geometric considerations and employing Snell's law of refraction. The expressions are not presented here.

\subsection{Strategy for sound speed estimation}

Having obtained the Doppler shifted frequencies (of an original frequency $\omega_{j}$ ) for different time instants measured in the receiver time frame, it is now possible to obtain the matrix $A_{j}^{d}$ in Eq. (3) and then the power

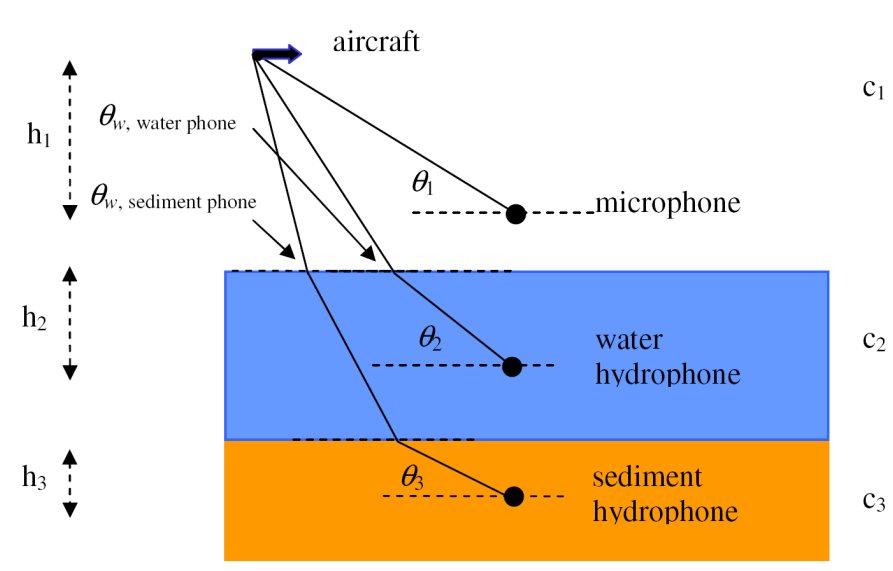

Figure 1: Schematic of measurement configuration.

spectrum of $P\left(\omega_{j}, c_{j}\right)$ from Eq. (6). We then repeat the same procedure but now for different discrete values of $c_{j}$ 's symmetrically distributed around the expected values (for example, when considering the microphone data, we let the sound speed change from $315 \mathrm{~m}$ to $375 \mathrm{~m}$ with a step size of $0.2 \mathrm{~m}$ ). This results in a power spectrum density function given at discrete sound speeds. In principle the sound speed at which this power spectrum achieves its maximum value is considered as the correct sound speed. We now consider a new frequency $\omega_{j}$. The same procedure is repeated to obtain yet a new sound speed for this frequency.

There are two issues concerning this strategy that we need to deal with. The first issue is that the frequencies $\omega_{j}$ of the harmonic functions are only ideally known. In practice, it is impossible to specify an individual value for the exact propeller frequency and its harmonics. We then have to be satisfied with a set of high-power frequencies around the original frequencies. We have considered the whole range of frequencies from $1 \mathrm{~Hz}$ to 1000 $\mathrm{Hz}$ with step size of $0.1 \mathrm{~Hz}$. For each propeller frequency (and its harmonics) a set of frequencies (those that have the largest mean powers) is then considered. For each of these close frequencies a sound speed is obtained (at the maximum power) and then the average is used as its final estimate.

The second point is that the power spectrum (as a function of the sound speed) does not have always only one clear peak, but sometimes a few peaks. Such a behavior is probable to occur due to external effects like e.g. multipath error. As a remedy, we suggest to fit a second order polynomial to the power spectrum and find its maximum. Figure 2 shows typical examples of one- and two-peak power spectra along with their second order polynomials fitted by the least-squares method. The peaks can be uniquely determined in this case.

\section{$3 \quad$ Results and discussions}

\subsection{Results on sound speed}

For testing the proposed approach, we have used two sample recordings made by Buckingham in 2003 on two different airplanes ([2]): the Cessna 172SP and the Diamond Star. The Cessna was flown at altitude of $244 \mathrm{~m}$ 

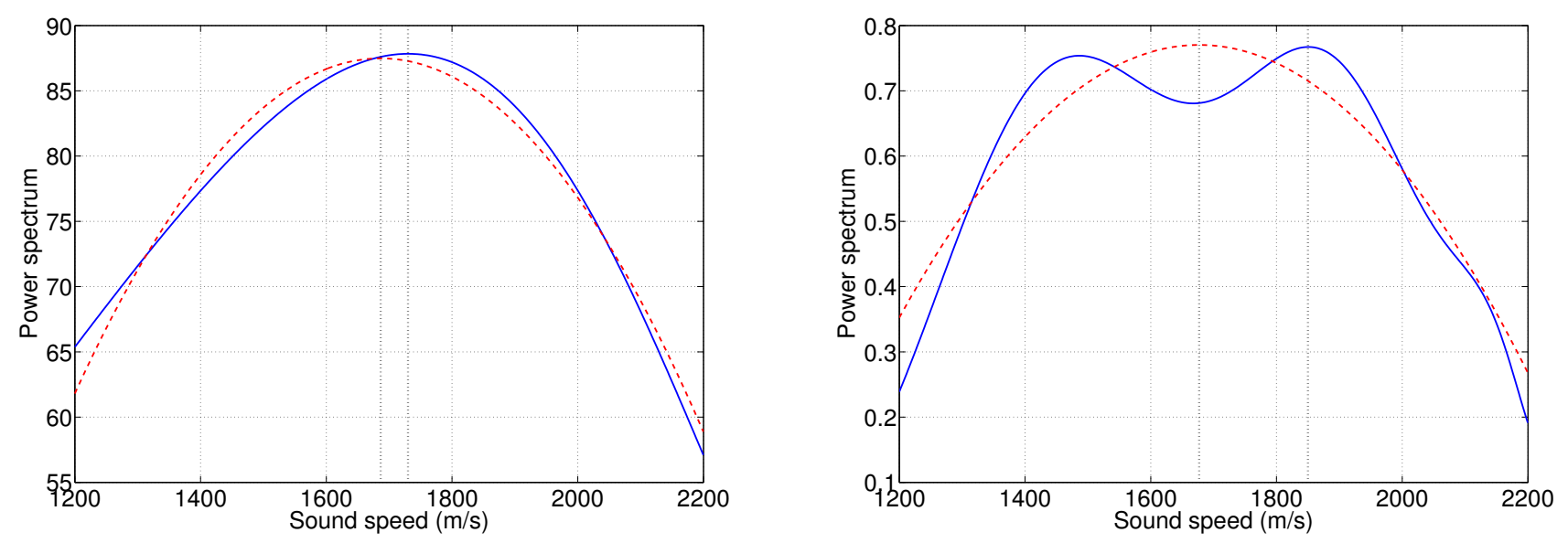

Figure 2: Power spectrum (solid curve) as a function of sound speed for two typical frequencies; one-peak spectrum (left), two-peak spectrum (right). Dashed curve shows the corresponding second order polynomial fitted by leastsquares. Dotted line indicates sound speed at which the power is maximum.

and the Diamond Star was flown at $61 \mathrm{~m}$. All recordings were sampled at $3200 \mathrm{~Hz}$. The depth of water as well as the depth of the hydrophone is considered to be $10 \mathrm{~m}$, and the depth of the sediment hydrophone is $0.75 \mathrm{~m}$.

For our analysis we let the frequency $f_{j}=\omega_{j} / 2 \pi$ changes from $0.1 \mathrm{~Hz}$ to $1000 \mathrm{~Hz}$ with the step size of 0.1 $\mathrm{Hz}$ (10,000 individual frequencies all together). Also, the sound speeds in the air, water, and sediment change from 315,1000 , and $1200 \mathrm{~m} / \mathrm{s}$ to 375,2000 , and 2200 $\mathrm{m} / \mathrm{s}$ with the step size of $0.2,2$, and $2 \mathrm{~m} / \mathrm{s}$, respectively. Then, the final sound speed estimates are obtained based on the average of the individual high-power estimates.

The results for the sound speed estimates in the air and water for the Cessna 172SP are given in Fig. 3 (top frames) using the strategy mentioned above. The sound speed does not seem to be dependent on the frequency, in agreement with the assumption that air and water are non-dispersive media. The observed independence of frequency gives confidence in our data processing method. For the sediment, however, a clear reduction of the sound speed at lower frequencies is observed (see top frames in Fig. 4; Cessna 172SP (left) and Diamond Star (right)). This is verified using the statistical hypothesis testing procedure given in the next subsection.

\subsection{Frequency dependent behavior}

To test a possible dependence of the estimated sound speed on the frequency, a set of polynomials will be fitted to the data given at different frequencies. For this purpose, consider the sound speeds $s=\left[s_{1}, \ldots, s_{m}\right]^{T}$ at frequencies $f=\left[f_{1}, \ldots, f_{m}\right]^{T}$ (here $m=23$ ). The problem now is to find the appropriate polynomial degree using the following null and alternative hypothesis:

$$
\mathrm{H}_{o}: \mathrm{E}(s)=A x, \text { versus } \mathrm{H}_{a}: \mathrm{E}(s)=A x+a_{i} x_{i}
$$

where $A$ is an $m \times n$ design matrix, $x$ is the $n$-vector of unknowns, and $a_{i}$ is an $m$-vector with its jth entry equal to $f_{j}$ to the power $i$. To start, we put the degree $i=1$ under the alternative hypothesis and the design matrix $A=a_{0}$ is a vector of ones - the summation vector. The test statistic of the preceding hypotheses is given as [5]

$$
w=\frac{e_{0}^{T} a_{i}}{\sigma_{a}\left(a_{i}^{T} P_{A}^{\perp} a_{i}\right)^{1 / 2}}
$$

where $e_{0}=P_{A}^{\perp} s$ is the least-squares residuals under the null hypothesis, $P_{A}^{\perp}=I-A\left(A^{T} A\right)^{-1} A$ is an orthogonal projector, and $\sigma_{a}^{2}=e_{a}^{T} e_{a} /(m-n-1)$ is the variance component under the alternative hypothesis $\left(e_{a}=P_{\left[A a_{i}\right]}^{\perp} s\right.$ is the least-squares residuals under the alternative hypothesis). Under $\mathrm{H}_{o}$, the test statistic has a t-distribution with $m-n$ degrees of freedom, i.e.

$$
w \sim \mathrm{t}(m-n)
$$

If the null hypothesis is rejected, we modify the design matrix $A \leftarrow\left[A a_{i}\right]$, increase $i$ by one step, and then perform the same procedure for testing yet another degree of the polynomial.

This method is now applied to the estimated sound speeds in air, water, and sediment to evaluate their dependence on the frequency. The results are given in Figs. 3 and 4 . In each figure, the test statistics as well as their critical values (type I error $\alpha=0.001$ ) are given for the first 10 polynomials (bottom frame) and the best fit, based on the results of the hypotheses testing, is also illustrated (top frame).

For the air and water (Fig. 3), the sound speed does not seem to be dependent, in a linear or quadratic form, on the frequency (the test statistic is below the critical value). Except for a periodic-like behavior of the sound speed (expressed by a sixth order polynomial), which is likely due to multipath effect, the sound speed remains independent of the frequency. This however does not hold for the marine sediment. The sound speed is best described by a second order polynomial (Fig. 4). This therefore statistically confirms that the sediment sound speed is indeed frequency-dependent and is well described by a quadratic decrease at lower frequencies.

\subsection{Comparison with model predictions}

From Fig. 5, a variation of sound speed with frequency can be seen. This behavior is denoted by dispersion. 

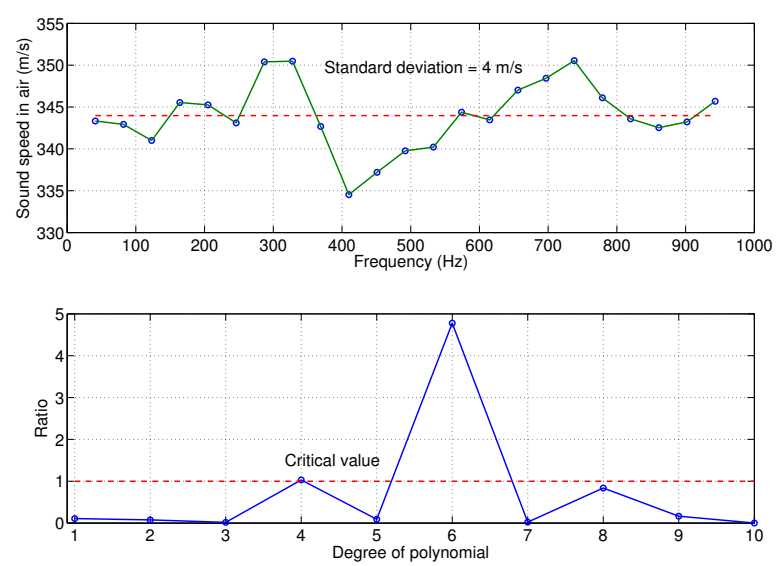
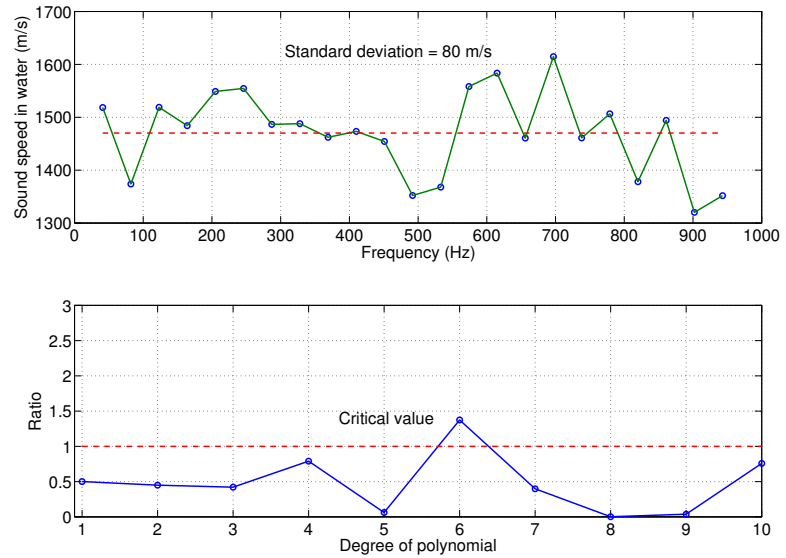

Figure 3: Sound speeds estimates (top frame) in air (left) and in water (right) as well as their best polynomial fit (dashed line) using the results of hypothesis testing; normalized test statistics (bottom frame), i.e. ratio of test statistics to their corresponding critical values, versus the critical value (dashed line which is set to one for normalized statistics) to test polynomials up to degree 10; Cessna 172SP test.
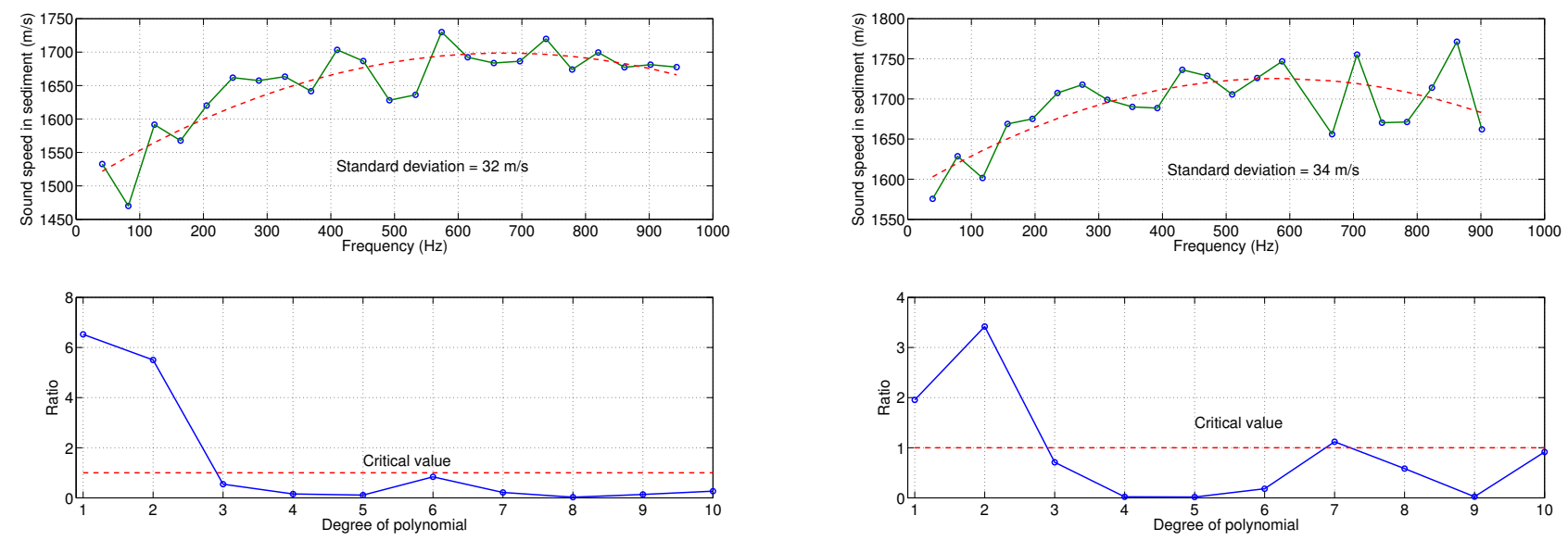

Figure 4: Sound speeds estimates (top frame) in marine sediment as well as their best polynomial fit (dashed line) using the results of hypothesis testing, Cessna 172SP test (left) and Dimond star test (right); normalized test statistics (bottom frame), i.e. ratio of test statistics to their corresponding critical values, versus the critical value (dashed line which is set to one for normalized statistics) to test polynomials up to degree 10 .

In [3] a model is presented for predicting the sediment sound speed dispersion behavior as a function of sediment material properties.

The model predicts the sound speed as a function of three parameters, i.e., $c_{0}, c_{\infty}$ and $f_{T}$. Here, $c_{0}$ is the sound speed at low frequencies, whereas $c_{\infty}$ is the limiting high frequency sound speed value. The $f_{T}$ is the transition frequency, which separates the low-frequency and high-frequency domain. In [3] expressions are presented that relate these three parameters to eight geotechnical parameters:

- Density of mineral grains $\rho_{s}$;

- Bulk modulus of mineral grains $K_{r}$;

- Density of pore fluid $\rho_{f}$;

- Bulk modulus of pore fluid $K_{f}$;

- Viscosity of pore fluid $\eta$;

- Porosity $\beta$;
- permeability $\kappa$;

- Tortuosity $\alpha$.

Based on values for the above parameters, the model described in [3] predicts the dispersion curve. Parameters $\rho_{s}, K_{r}, \rho_{f}$, and $K_{f}$ have the same values for all sediments, being $2650 \mathrm{~kg} / \mathrm{m} 3,3.6 \times 10^{10} \mathrm{~Pa}, 1000 \mathrm{~kg} / \mathrm{m}^{3}$ and $2.25 \times 10^{9} \mathrm{~Pa}$, respectively. Pore fluid viscosity typically has a value of $0.001 \mathrm{~kg} / \mathrm{ms}$. Porosity $\beta$ can take values ranging from 0.37 to 0.85 . The permeability of unconsolidated sediments shows a large spread, ranging from $10^{-10}$ to $10^{-13} \mathrm{~m}^{2}$. Tortuosity typically takes values in between 1 and 3 .

The acoustic measurements, described in the previous sections, were taken in a sandy environment. For sandy environments porosities typically take values from 40 to $50 \%$. Figure 5 shows a comparison between the modeled dispersion curve with the estimated sound speeds. As mentioned above, the majority of the sediment parameters have fixed values. Porosity, permeabil- 

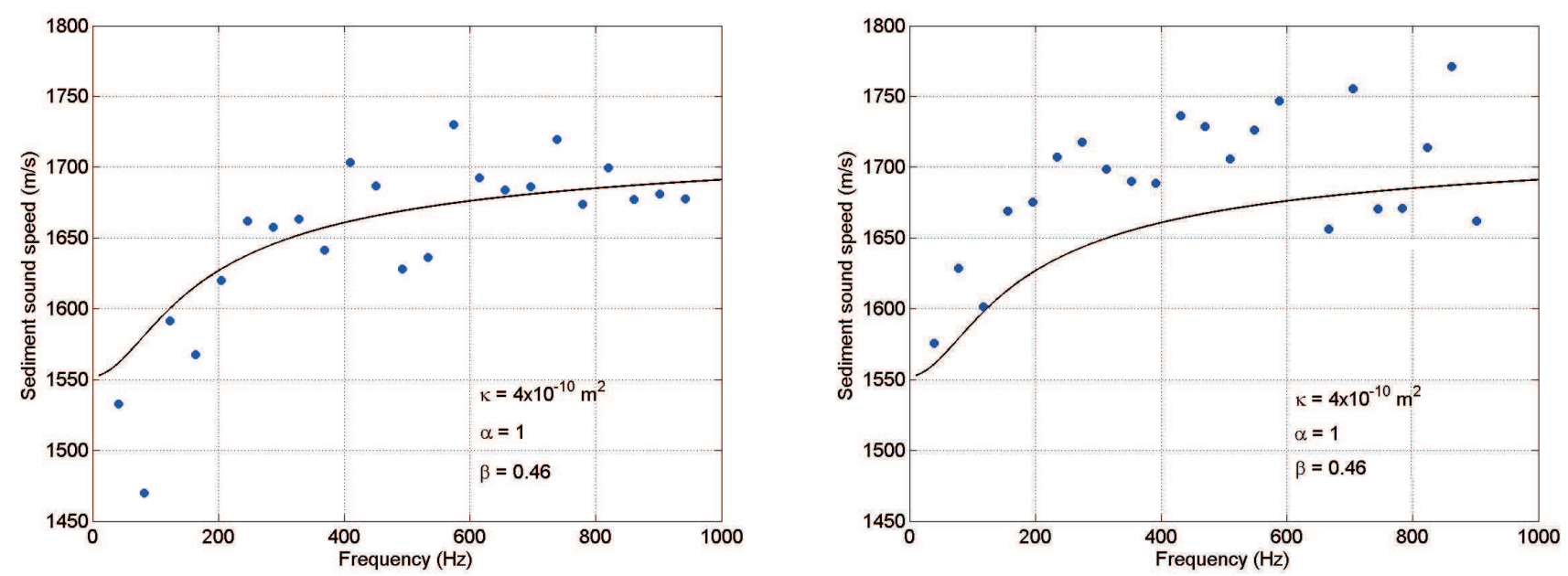

Figure 5: Comparison of predicted sound velocity dispersion curve (black solid line), with measured dispersion curves; Cessna 172SP test (left), Diamond Star test (right).

ity and tortuosity, however, can differ significantly from sediment to sediment. Here, values of 0.46 for porosity, 1 for tortuosity and $4 \times 10^{-10} \mathrm{~m}^{2}$ for permeability have been selected to obtain a good match between model output and measurements. A slight difference between the model and the estimated values (Fig. 5 right) is likely due to the errors in the parameters which were kept fixed in the analysis.

\section{Concluding remarks}

This contribution presented a new method to estimate the speed of sound in air, water, and marine sediment. The propeller noise harmonics (of low frequency) of a light aircraft, which exhibit a noticeable Doppler frequency shift, were used to assess the role of different sound speeds in the construction of the power spectrum density developed in the least-squares framework. The sound speed that maximizes the power spectrum is considered to be an estimate for the sound speed in the medium. The method can provide a sound speed for each of the propeller harmonics.

Statistical tests confirm that the sound speed in the marine sediment is dependent on the frequency; a reduction at lower frequencies is clearly observed. These findings also confirm the dispersions predicted from the theoretical models. The method needs to be tested on different data sets to see whether indeed such behavior start to stand out. Also, the future work should consider the other theoretical approaches such as the grainshearing theory presented recently by [6], which is more appropriate for marine sediments.

The absolute values of the estimates for the sound speeds depend mainly on the correct values of the arguments involved. Here, the parameters $v, h_{1}, h_{2}$, and $h_{3}$ were kept fixed. There however exist approximate values for these parameters. In future experiments, we recommend to measure them as precise as possible. Perhaps GPS geodetic receivers can be employed to measure the position (and also the velocity) of the aircraft with a centimeter level of precision in a differential mode.

\section{References}

[1] M. J. Buckingham, E. M. Giddens, F. Simonet, and T. R. Hahn. Propeller noise from a light aircraft for low-frequency measurements of the speed of sound in a marine sediment. Journal of Computational Acoustics, 10(4):445-464, 2002.

[2] Aircraft Sound. 2003. http://extreme.ucsd.edu/ Research/ AircraftSound/ AircraftSound.html.

[3] M. J. Buckingham. A three-parameter dispersion relationship for Biot's fast compressional wave in a marine sediment. Journal of the Acoustical Society of America, 116(2):769-776, 2004.

[4] P. J. G. Teunissen. Adjustment theory: an introduction. Delft University Press, Website http://www.vssd.nl, 2000. Series on Mathematical Geodesy and Positioning.

[5] P. J. G. Teunissen, D. G. Simons, and C. C. J. M. Tiberius. Probability and observation theory. Delft University, Faculty of Aerospace Engineering, Delft University of Technology, 2005. Lecture notes AE2E01.

[6] M. J. Buckingham. On pore-fluid viscosity and the wave properties of saturated granular materials including marine sediment. Journal of the Acoustical Society of America, 122(3):1486-1501, 2007. 